\title{
Multibeam second-harmonic generation by spatiotemporal shaping of femtosecond pulses
}

\author{
Raúl Martínez-Cuenca, ${ }^{1,2, *}$ Omel Mendoza-Yero, ${ }^{1,2}$ Benjamín Alonso, ${ }^{3}$ Íñigo Juan Sola, ${ }^{3}$ \\ Gladys Mínguez-Vega, ${ }^{1,2}$ and Jesús Lancis ${ }^{1,2}$ \\ ${ }^{1}$ GROC UJI, Departament de Física, Universitat Jaume I, 12080 Castelló, Spain \\ ${ }^{2}$ Institut de Noves Tecnologies de la Imatge (INIT), Universitat Jaume I, 12080 Castelló, Spain \\ ${ }^{3}$ Departamento de Física Aplicada, Universidad de Salamanca, E37008 Salamanca, Spain \\ *Corresponding author: rcuenca@fca.uji.es
}

Received October 20, 2011; revised December 22, 2011; accepted December 29, 2011; posted January 3, 2012 (Doc. ID 156757); published February 29, 2012

\begin{abstract}
We present a technique for efficient generation of the second-harmonic signal at several points of a nonlinear crystal simultaneously. Multispot operation is performed by using a diffractive optical element that splits the near-infrared light of a mode-locked Ti:sapphire laser into an arbitrary array of beams that are transformed into an array of foci at the nonlinear crystal. We show that, for pulse temporal durations under $100 \mathrm{fs}$, spatiotemporal shaping of the pulse is mandatory to overcome chromatic dispersion effects that spread both in space and time the foci showing a reduced peak intensity that prevents nonlinear phenomena. We experimentally demonstrate arbitrary irradiance patterns for the second-harmonic signal consisting of more than 100 spots with a multipass amplifier delivering $28 \mathrm{fs,}$ $0.8 \mathrm{~mJ}$ pulses at $1 \mathrm{kHz}$ repetition rate. () 2012 Optical Society of America
\end{abstract}

OCIS codes: $\quad 090.1970,320.7100,320.7110$.

Current high-gain femtosecond amplifiers running at kilohertz repetition rate provide output pulse energies at the millijoule level that overpass by a huge amount the required energy for some applications. For instance, the pulse energies used at the low fluence regime for surface micro- and nanostructuring of materials (e.g., metals and semiconductors) are often $<10 \mu \mathrm{J}$. Similar values are required for pulse processing of dielectric solids (fused silica) or liquid resins [1]. Also, pulsed Ti:sapphire lasers offer abundant light for multiphoton excitation in nonlinear microscopy with $90-95 \%$ of the light discarded. The use of multiple beams in parallel to take advantage of the full power of the amplifier system has been proposed and demonstrated in fields as material processing [2]-[8] and multiphoton microscopy [9-11]. Such methods rely on the use of diffractive optical elements (DOEs) or microlens arrays to divide the laser beam into several beamlets that, after focusing, scan the sample simultaneously. DOEs are usually the choice as they allow dynamic codification.

The use of DOEs however is problematic for laser pulses in the femtosecond regime. Several authors have reported an increase of the eccentricity of the focused spots with the transverse distance at the irradiation plane that is attributed to the finite bandwidth of the laser source $[3,7,9]$. For example a 100 fs pulse at center wavelength of $790 \mathrm{~nm}$ has a bandwidth of approximately $15 \mathrm{~nm}$ or $1.9 \%$ of the center wavelength. This situation is even worse for shorter pulse durations. More interestingly, the pulse is also stretched in time as a result of the propagation time difference between pulses crossing the DOE at different transverse positions (angular dispersion). This effect cannot be precompensated with the chirping of the original pulse as group-velocitydispersion broadening. As a result, there is an inherent drop of the peak intensity inhomogeneously distributed over the working area. This fact prevents nonlinear interactions in outer regions from the axis. Note that, for instance, the second-harmonic ( $\mathrm{SH})$ signal increases inversely with pulse duration [12].
The bandwidth limitation is a characteristic of all diffractive optics when used with a broadband source. We consider a simple picture where a low-frequency diffraction grating splits the pulsed laser beam into several diffracted beamlets. The beamlets are gathered by an achromatic objective and focused to an array of light spots at its back focal plane. As a result of the angular dispersion of the grating, each spectral component is focused at a different transverse position, which results in an elongated light spot, except for the zero-order. Also, the decrease in the available bandwidth at each maximum due to the lateral walk-off of the different spectral components results in the temporal stretching of the pulse. Several attempts were done to compensate for the chromatic distortion [13]-[18]. In 13 a diffractive lens pair was proposed. The key point is the strong chromatic aberration of diffractive lenses.

In this Letter we provide experimental evidence of the energy spreading both in space and particularly in time of the array of foci generated from a DOE illuminated with a femtosecond laser pulse. Spatially and temporally resolved measurement of the complex spatiotemporal light field at the multifocal pattern was carried out by using a recently reported technique that is based on the spatiotemporal amplitude and phase reconstruction by Fourier transform of the interference spectra of the optical beams (STARFISH) $[19,20]$. Also, the ability of the diffractive lens pair to compensate for energy spreading both in space and time of the foci is experimentally tested. Finally, we demonstrate multifocal SH generation inside a BBO crystal by use of the compensating module.

Figure 1 shows the proposed setup. A mode-locked Ti:sapphire source (Femtosource, Femtolaser) provides the excitation source. The temporal width of the pulses is 28 fs amplitude FWHM and the central wavelength of the corresponding spectra is $\lambda_{0}=800 \mathrm{~nm}$ with a bandwidth of approximately $80 \mathrm{~nm}$. The maximum energy per pulse is $0.8 \mathrm{~mJ}$ at a $1 \mathrm{kHz}$ repetition rate. The average energy per pulse was continuously adjusted with a half-wave plate and a polarizer and the beam width was fixed using 


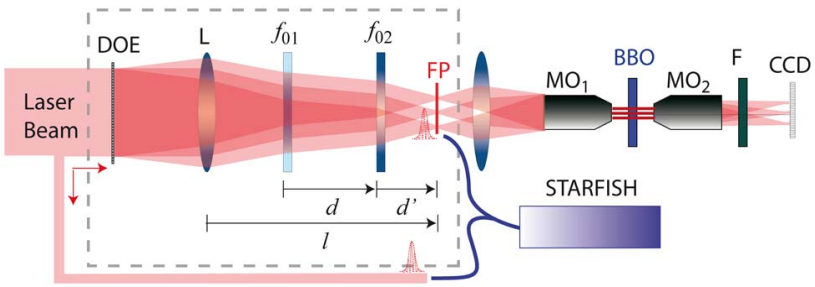

Fig. 1. (Color online) Optical setup with the DCM for beam delivery.

a reflective beam expander. The output from the Ti:sapphire first passes through a pair of fused silica Brewster prisms. The prism pair introduces negative dispersion to later compensate the group delay dispersion (GDD) in the beam delivery path until the observation plane. The pulsed laser beam (PLB) impinges onto a Fourier DOE that, by virtue of the beam delivery optics inside the dashed box, generates multiple foci onto its back focal plane (FP).

The beam delivery optics (BDO) is designed as a dispersion compensating module (DCM) to provide an output irradiance distribution corresponding to the nearly wavelength-independent Fourier transform of the DOE amplitude at its back focal plane. More specifically, see dashed box in Fig. 1, beam delivery is accomplished by means of a lens achromat $L$ (with focal length $f$ ) coupled to a diffractive lens pair $\mathrm{DL}_{1} \mathrm{DL}_{2}$ as was introduced in $\underline{13}$. The focal lengths of $\mathrm{DL}_{1}$ and $\mathrm{DL}_{2}$ for the center wavelength are denoted by $f_{o, 1}$ and $f_{o, 2}$, respectively. Initially, the system acts as a Fourier transformer for the center wavelength, i.e. the field at FP is the Fourier transform of the field in the DOE plane for the center wavelength. In a second step, we force the design to ensure that the center of every spectral spot corresponding to the same diffraction order coalesces in a single point for all the spectral components and that every ray impinging onto the DOE has an identical arrival time at the back focal plane. Although exact compensation is not possible, we impose a first-order or achromatic correction that leads to the geometrical constraints, $l=f, d^{2}=$ $-f_{o, 1}, f_{o, 2}$, and $d^{\prime}=-d^{2} /\left(d+2 f_{o, 1}\right)$. As a result, the actual foci provided by the DCM shows a slight spatial and temporal stretching with respect to the otherwise ideal transform-limited spots [13]. To compare the optical features of our proposal with a conventional setup (without DCM) we simply remove the diffractive lens pair, and displace the achromatic lens $L$ just to locate its back focal plane at FP.

Initially, multiple foci are created by use of a Ronchi grating as a DOE that has the property to distribute the incident radiation in distinct diffraction orders at the output FP. The position of the diffracted orders is determined by the period of the grating.

We performed the measurement of the spatiotemporal light distribution of the different foci at FP by using STARFISH [19]. This reconstruction technique consists of spatially resolved spectral interferometry using a fiber optic coupler as interferometer. Here, the light from the ultrafast laser source is split in two arms: the reference and the test beam. The reference and the test beam are collected into the fiber input ports. The test arm spatially scans the transverse profile at the FP of the BDO and the reference arm controls the relative delay between the pulses required for the interferometry (between 2-3 ps in the experimental conditions of the present study). For each transverse position, both pulses are combined inside the fiber coupler and leave it through the output common port that is directly connected to a standard spectrometer where the interference spectra are measured. Also, the reference beam is not scanned and its spectral phase is obtained on-axis just by means of a conventional spectral phase interferometry measurement (SPIDER). From the spectral interference, the spectral phase and amplitude at each point are obtained by digital processing using conventional algorithms and finally the temporal profile is recovered by Fourier transformation.

For the experimental implementation we constructed the optical setup in Fig. 1 with $f=200 \mathrm{~mm}, f_{o, 1}=$ $-150 \mathrm{~mm}$, and $f_{o, 2}=150 \mathrm{~mm}$ and a numerical aperture (NA) of 0.02 and used a Ronchi grating with fundamental frequency $\nu_{s}=11.8 \mathrm{lp} / \mathrm{mm}$. Results are shown in Fig. 2 where the spatiotemporal light intensity at the output plane FP is measured with STARFISH. To show dispersive effects, the transverse coordinate at FP normal to the grating ruling is shown at the horizontal axis. The scan is performed through the line with the maximum irradiance for the diffractive foci. The temporal coordinate is displayed at the vertical axis. Right and left columns show the spatiotemporal light distribution corresponding to the $0,+1,+2$, and +3 diffraction orders with and without DCM, respectively. Each plot is centered around the position of the spectral spot for the center wavelength $\lambda_{0}$. For the plot, light intensity was normalized to the same value for the different maxima. The capability of the DCM to compensate dispersive stretching is noticeable at increasing frequency components of the grating that are located at outer regions of FP. The root-mean-square (RMS) width for both spatial and temporal coordinates

Spatio-temporal irradiance distributions
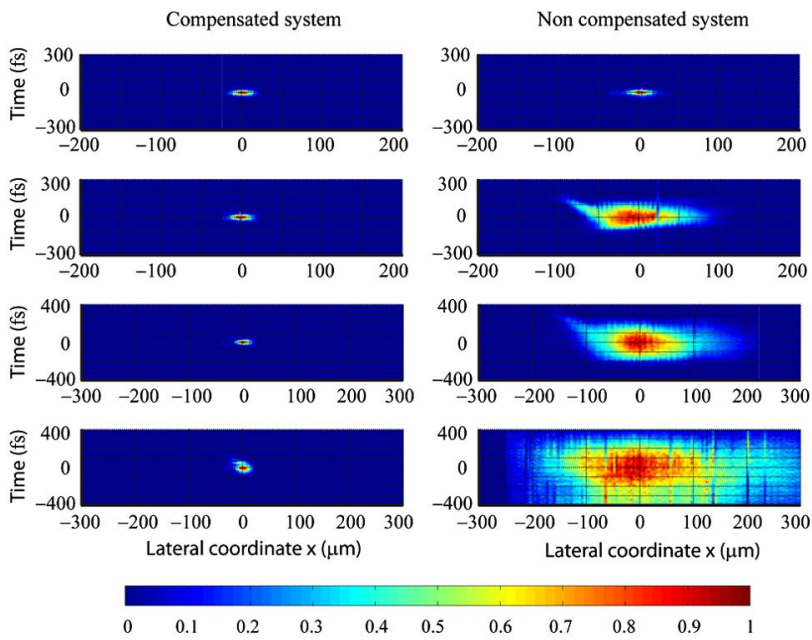

Fig. 2. (Color online) Normalized spatiotemporal light intensity after low NA focusing of the beamlets coming from a DOE $(0,+1,+2$, and +3 diffraction orders from top to bottom) with (left colum) and without (right colum) DCM. Measurements were captured using STARFISH [19]. The maximum frequency component, for the third diffraction order, is $35.4 \mathrm{lp} / \mathrm{mm}$. 
of the compensated foci (right column) is $2 \sigma_{x}=18.0 \mu \mathrm{m}$ and $2 \sigma_{t}=26.8 \mathrm{fs}$ ( 0 order), $2 \sigma_{x}=18.6 \mu \mathrm{m}$ and $2 \sigma_{t}=$ $27.5 \mathrm{fs}\left(+1\right.$ order), $2 \sigma_{x}=18.7 \mu \mathrm{m}$ and $2 \sigma_{t}=31.9 \mathrm{fs}$ $(+2$ order $)$ and $2 \sigma_{x}=19.3 \mu \mathrm{m}$ and $2 \sigma_{t}=63.2 \mathrm{fs}(+3$ order). The widths remain nearly constant up to frequency components of about $30 \mathrm{lp} / \mathrm{mm}$. We also notice some ripples in the temporal profile that are due to noncompensated third-order dispersion (TOD) in the glass components. Also radial GDD effects are apparent for the higher spatial frequencies [13]. The foci generated without DCM suffer from a higher spatiotemporal stretching (even one order of magnitude for the higher spatial fequencies). Specifically only $10 \%, 2.5 \%$, and $0.5 \%$ of the available peak-power for the compensated foci is delivered to the spatiotemporal smeared diffraction orders $+1,+2$, and +3 , respectively. This fact limits the capability to generate simultaneous $\mathrm{SH}$ with the DOE.

Finally, we experimentally demonstrate arbitrary irradiance patterns at the SH signal. To excite the SH signal we used an additional lens to cover the full aperture of a $10 \times$ microscope objective $M O_{1}$ (160 mm conjugated, 0.25 numerical aperture) to make a reduced image of the diffractive foci at the sample, in our case a BBO crystal with $20 \mu \mathrm{m}$ thickness. Additional relay optics introduces extra GDD and TOD. Although GDD was compensated by tuning the prism pair to maximize the SH yield, TOD prevents from achieving a transform-limited 28 fs pulse at the sample. Spatial resolution is fixed by the numerical aperture (NA) of the microscope and is estimated to be of $1.53 \mathrm{~mm}$. Field curvature of the microscope objective increases slightly the beam diameter in the low spatial frequency region while harmonic components higher than $20 \mathrm{lp} / \mathrm{mm}$ in our optical setup are more seriously affected by the residual chromatism. To observe the $\mathrm{SH}$ signal, the BBO crystal is imaged onto a conventional CCD sensor (Ueye UI-1540M) by means of a 10× microscope objective $\mathrm{MO}_{2}$ also conjugated at $160 \mathrm{~mm}$. We placed a suited filter F2 (BG39-Schott crystal) before the CCD camera to absorb the infrared radiation at $800 \mathrm{~nm}$ coming directly from the laser (not from the second harmonic signal). We used a Fourier DOE where the complex Fourier transform of a "smiling face" was codified in amplitude as an off-axis binary computer generated hologram. The computer reconstruction of the Fourier DOE shows a set of more than 100 diffractive foci. The spatial spectrum of the sample spreads from around $5 \mathrm{lp} / \mathrm{mm}$ to about $40 \mathrm{lp} / \mathrm{mm}$. Irradiance uniformity of the foci is estimated to be of $65 \%$. Results of the recorded SH signal are shown in Fig. 3 without [Fig. 3(a))] and with [Fig. 3(b)] the DCM. For the uncompensated system only the lower spatial frequencies are able to excite SH signal at the incoming light energy.

In summary, we have demonstrated efficient generation of the SH signal at several points of a nonlinear crystal using broadband light pulses and the focused beamlets coming from a DOE. The system incorporates a DCM to increase the light peak-power at the foci enhancing nonlinear phenomena.

This work was supported by the Spanish Ministerio de Ciencia e Innovación (MICINN) and FEDER through the
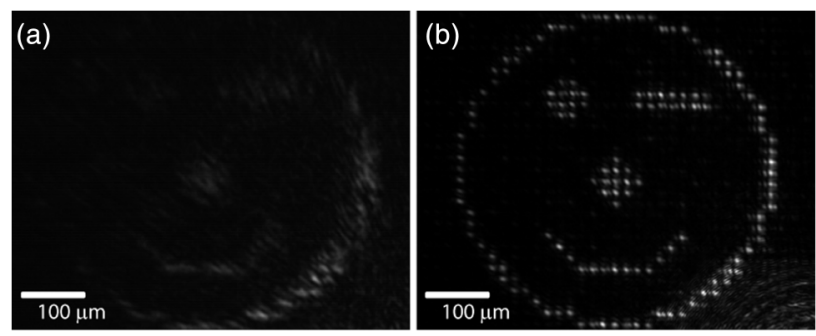

Fig. 3. SH signal from a BBO crystal illuminated with a multispot pattern originated from a DOE (a) without and (b) with the DCM.

projects FIS2010-15746, FIS2009-09522, and SAUUL (CSD2007-00013) and the Fundació Caixa Castelló (P11B2010-26). The authors are grateful to the Serveis Centrals d'Intrumentació Científica of the Universitat Jaume I and the Centro de Láseres Pulsados, Salamanca. We are indebted to Professor Jürgen Jahns for providing us with the diffractive lenses.

\section{References}

1. S. Nolte, in Ultrafast Optics: Technology and Application, M. E. Fermann, A. Galvanauskas, and G. Sucha, eds. (Marcel Dekker, 2003).

2. Y. Kuroiwa, N. Takeshima, Y. Narita, S. Tanaka, and K. Hirao, Opt. Express 12, 1908 (2004).

3. Y. Hayasaki, T. Sugimoto, A. Takita, and N. Nishida, Appl. Phys. Lett. 87, 031101 (2005).

4. C. Mauclair, G. Cheng, N. Huot, E. Audouard, A. Rosenfeld, I. V. Hertel, and R. Stoian, Opt. Express 17, 3531 (2009).

5. M. Sakakura, T. Sawano, Y. Shimotsuma, K. Miura, and K. Hirao, Opt. Express 18, 12136 (2010).

6. S. Hasegawa and Y. Hayasaki, Opt. Lett. 36, 2943 (2011).

7. Z. Kuang, W. Perrie, J. Leach, M. Sharp, S. P. Edwardson, M. Padgett, G. Dearden, and K. G. Watkins, Appl. Surf. Sci. 255, 2284 (2008).

8. K. Obata, J. Koch, U. Hinze, and B. N. Chichkov, Opt. Express 18, 17193 (2010).

9. A. H. Buist, M. Müller, J. Squer, and G. J. Brakenhoff, J. Microsc. 192, 217 (1998).

10. B. O. Watson, V. Nikolenko, and R. Yuste, Front. Neural Circuits 3, Article 6 (2008).

11. N. Ji, J. C. Magee, and E. Betzig, Nat. Methods 5, 197 (2008).

12. S. Tang, T. B. Ktasieva, Z. Chen, G. Tempea, and B. J. Tromberg, J. Biomed. Opt. 11, 02501 (2006).

13. G. Mínguez-Vega, J. Lancis, J. Caraquitena, V. TorresCompany, and P. Andrés, Opt. Lett. 31, 2631 (2006).

14. G. Mínguez-Vega, E. Tajahuerce, M. Fernández-Alonso, V. Climent, J. Lancis, J. Caraquitena, and P. Andrés, Opt. Express 15, 278 (2007).

15. J. Amako, K. Nagasaka, and N. Kazuhiro, Opt. Lett. 27, 969 (2002)

16. G. Li, C. Zhou, and E. Dai, J. Opt. Soc. Am. A 22, 767 (2005).

17. S. P. Veetil, H. Schimmel, and F. Wyrowski, J. Opt. Soc. Am. B 24, 2580 (2007).

18. K. Kimura, S. Hasegawa, and Y. Hayasaki, Opt. Lett. 35, 139 (2010).

19. B. Alonso, I. J. Sola, O. Varela, J. Hernández-Toro, C. Méndez, J. San Román, A. Zaïr, and L. Roso, J. Opt. Soc. Am. B 27, 933 (2010).

20. O. Mendoza-Yero, B. Alonso, O. Varela, G. Mínguez-Vega, I. J. Sola, J. Lancis, V. Climent, and L. Roso, Opt. Express 18, 20900 (2010). 